hautnah 2013 $12: 3$

DOI 10.1007/s12326-013-0048-2

(c) Springer-Verlag 2013

\author{
A.Sillaber \\ Springer-Verlag, Wien
}

\title{
hautnah im neuen Outfit
}

Patientenschulungsprogrammen und Büchern in Millionenauflage.

Damit Sie, unsere Leserinnen und Leser, noch mehr vom riesigen redaktionellen Think Tank profitieren, intensivieren wir die globale Zusammenarbeit aller Standorte von Springer Science+Business Media. Diese interne Vernetzung macht es möglich, in Zukunft bei allen wichtigen Kongressen durch unsere Fachredaktionen präsent zu sein - die Inhalte werden noch aktueller und internationaler.

\section{Immer aktuell}

Und von noch einer weiteren Entwicklung profitieren Sie: So wird auch Springer Wien noch stärker in die e.Aktivitäten des Verlages eingebunden. Die digitalen Varianten (Internet, Tablet, Smartphone, App) machen unser Informationsangebot in der Medizin zukünftig vollständig sie stehen gleichberechtigt neben unseren hochwertigen Printprodukten.

So sind die in hautnah publizierten Inhalte auch auf der Website von SpringerMedizin.at nachzulesen.

Das tagesaktuelle Portal SpringerMedizin.at bietet Ihnen Informationen zur neuesten medizinischen Fachliteratur, Berufspraxis, Gesundheitspolitik und Fortbildung. Registrieren Sie sich für den wöchentlichen Newsletter, wählen Sie Ihre Fachbereiche aus - und schon erhalten Sie punktgenau die Medizin-News, die Sie wirklich interessieren!

In diesem Sinne wünschen wir Ihnen ein informatives und kompaktes Lesevergnügen mit der Zeitschrift, die Sie in Händen halten!
Haben Sie Fragen oder Anregungen zu unserer neuen hautnah? Dann nehmen Sie Kontakt mit mir auf - ich freue mich auf Ihre Nachricht!

Ihr

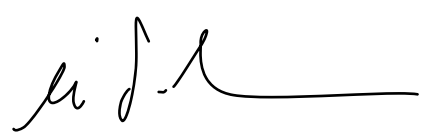

Dr. Alois Sillaber Springer-Verlag GmbH Managing Director alois.sillaber@springer.at 